\title{
High power THz-range Wave generation based on Transformation of Plasma Waves Pumped by High-current Relativistic Electron Beam
}

\author{
A.V. Arzhannikov ${ }^{1,2}$, V.V. Annenkov ${ }^{1,2}$, V.S. Burmasov ${ }^{1,2}$, I.A. Ivanov ${ }^{1,2}$, A.A. Kasatov ${ }^{1,2}$, \\ S.A. Kuznetsov ${ }^{1,2}$, M.A. Makarov ${ }^{1}$, K.I. Mekler ${ }^{1}$, S.V. Polosatkin ${ }^{1,2}$, V.V. Postupaev ${ }^{1,2}$, \\ A.F. Rovenskikh ${ }^{1}$, D.A. Samtsov ${ }^{1,2}$, S.L. Sinitsky ${ }^{1,2}$, V.F. Sklyarov ${ }^{1,2}$, V.D. Stepanov ${ }^{1,2}$, \\ I.V. Timofeev ${ }^{1,2}$, and E.P. Volchok ${ }^{1,2}$
}

${ }^{1}$ Budker Institute of Nuclear Physics Russian Academy of Science, Novosibirsk, Russia, arzhan1@ngs.ru ${ }^{2}$ Novosibirsk State University, Novosibirsk, 630090, Russia

\section{Introduction}

The beam-plasma interaction system allows one to generate high-power sub-mm waves (frequency interval $0.1 \div 1 \mathrm{THz}$ ) by usage of mechanism of plasma wave transformation [1]. This way gives possibility to achieve multi megawatt power with the promptly varying frequency that can be necessary for various practical applications. The original project of a sub-mm wave based on transformation of plasma waves pumped by a kA-current relativistic electron beam is developed at the GOL-PET facility. We present novel results on the study of mechanisms of sub-mm wave emission by the processes of plasma wave transformation in electromagnetic one in case of the strong beam-plasma interaction.

\section{Facility and results of experiments}

To study the conditions associated with the generation of electromagnetic radiation, the specialized facility GOL-PET was constructed. The GOL-PET facility consists of an open magnetic trap with a multiple-mirror or uniform magnetic field of mean value $\mathrm{B}=4.2 \mathrm{~T}$ and length $\mathrm{L}=2.4 \mathrm{~m}$ between the end mirrors, see Fig.1. The end mirrors have a strong field up to $\mathrm{B}=8 \mathrm{~T}$. A plasma column with the density $\mathrm{n}_{\mathrm{e}} \approx$ $(0.2 \div 5) \times 10^{15} \mathrm{~cm}^{-3}$ and a diameter of $7 \mathrm{~cm}$ is created by a longitudinal high-current discharge. U-2 accelerator producing a high current relativistic electron beam (REB) is mounted at one end of the trap. It produces the beam with the current $\mathrm{I} \sim 10 \mathrm{kA}$, the pulse duration $6 \mu$ s at the electron kinetic energy $\mathrm{E}_{\mathrm{e}} \approx 0.8$ $\mathrm{MeV}$. The REB is injected into the end of the plasma column and where the beam diameter is $4 \mathrm{~cm}$ at the magnetic field $4 \mathrm{~T}$. The radial profile of the plasma density is measured in 9-dots over the column diameter at a distance $\mathrm{z}=0.83 \mathrm{~m}$ from the entrance mirror by diagnostics based on Thomson scattering. Dynamics of the average plasma density is measured by Michelson interferometer at $\mathrm{z}=1.16 \mathrm{~m}$. Pulsed current transformers measure the electron beam current at different axial points. Electron energy of REB is determined according with accelerator voltage in the U2 diode. The transfer of energy from REB to plasma is calculated from results of measurements of diamagnetism of the plasma column. The 8-channel sub-mm polychromator, as well as a number of single detectors with bandpass filters at their entrance are utilized to study properties of radiation emitted by plasma [2].

Previous experiments showed that the changes of the power value of the emitted electromagnetic waves correlated with the variation in the efficiency of plas- ma heating by the electron beam. [1-3]. In a previous series of experiments on the simultaneous registration of radiation power emitted along the axis of the plasma column and in its perpendicular direction conducted at varying the plasma density from $10^{14}$ up to $10^{15}$ $\mathrm{cm}^{-3}$, we observed that the emission in the transverse direction was only at relatively low plasma densities (ne $<5 \times 10^{14} \mathrm{~cm}^{-3}$ ). For higher value of plasma density, the emission in the band above $300 \mathrm{GHz}$ was observed along the axis of the device only. Just as in the earlier experiments on the GOL-3, the EM-wave emission mainly exists simultaneously with the increasing of the plasma diamagnetism.

A series of experiments for measuring the temporal dynamics of radiation spectra emitted along the axis carried out for the plasma density higher than $5 \times 10^{14} \mathrm{~cm}^{-3}$. It was done by the 8 -channel polychromator with semiconductor diodes for the frequency interval from 0.1 up to $0.5 \mathrm{THz}$. For the interval $0.5 \div 0.9 \mathrm{THz}$ the measurements were done by single plasmonic detectors and an additional 2-channel system of cryogenic sensors. Experiments showed that at plasma density $5 \times 10^{14} \div 10^{15} \mathrm{~cm}^{-3}$ the spectral composition of the emission along the axis of the plasma column depended on radial gradient of the plasma density $[2,3]$. The increase of the gradient caused to strongly increasing the spectral power density in the frequency band $150 \div 300 \mathrm{GHz}$. An example of signals from various sensors for one of the pulses of the GOL-PET facility (\#1945) in case of the strong plasma density gradient (greater than $10^{15} \mathrm{~cm}^{-4}$ ) is presented in Fig. 2. The signal of the spectral power density from the diodes in the interval $150-300 \mathrm{GHz}$ is dumped in 50 times for presenting here. Taking into account, this dumping factor we can say that the power in the spectral interval $150 \div 300 \mathrm{GHz}$ is 25 $\mathrm{kW} /\left(\mathrm{sr} \cdot \mathrm{cm}^{2}\right)$ and the spectral power density is greater than its value in the interval $400 \div 800 \mathrm{GHz}$ approximately in two orders of the value.

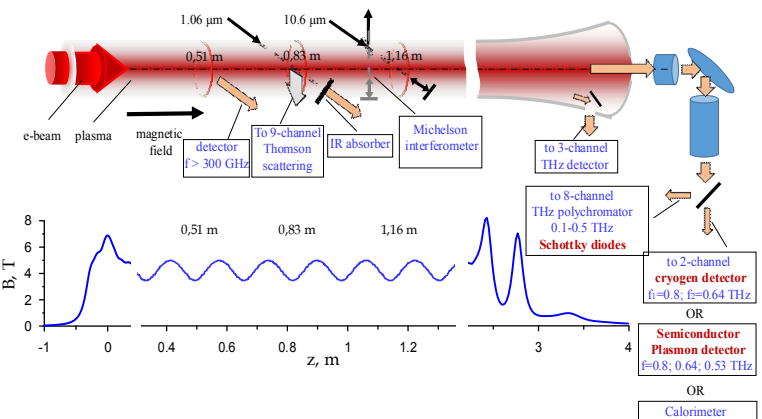

Fig. 1. Schematic of experiments at the GOL-PET facility 

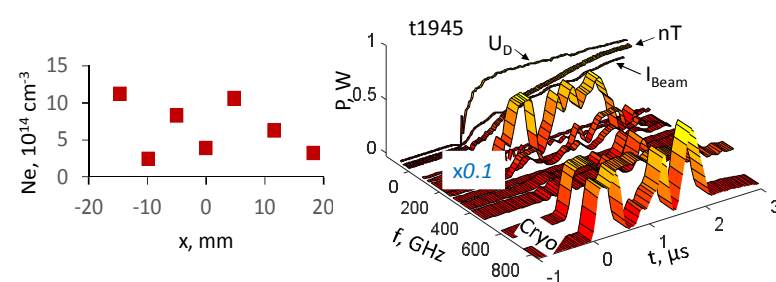

Fig. 2. Results of measurements in pulse \#1945 at the GOLPET facility. Left - plasma density distribution from 8channel Tomson scattering system; right - signals from various sensors. $U_{D}$ - diode voltage of the accelerator $(\mathrm{MeV})$, IBeam - beam current (10 kA), nT - plasma pressure from diamagnetic loop, other signals - radiation power in frequency selective detectors in Watts.
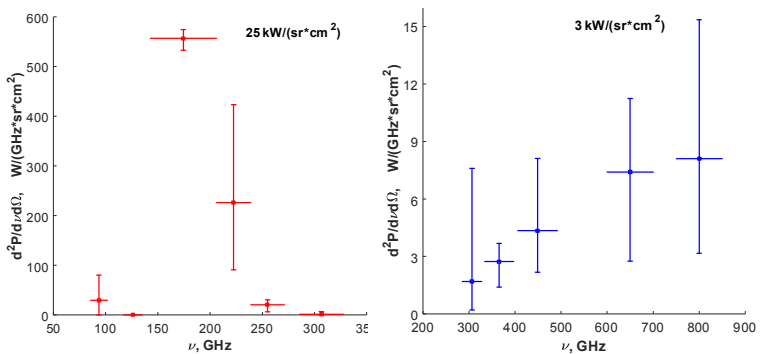

Fig. 3. Spectral composition of the plasma emission along the axis in case of strong plasma density gradient in transverse direction

Short analysis of measurement results The signals in the frequency band $125 \div 250 \mathrm{GHz}$ are interpreted as radiation near the plasma frequency, Fig. 3 (spectral band in left side of the picture). Such radiation can be produced through the linear mode conversion of upper-hybrid branch of plasma oscillations in region with plasma density gradients [4]. The observed width of the spectral line corresponds to the limited range of plasma density (3-6) $\times 10^{14} \mathrm{~cm}^{-3}$. It means that intense beam-driven oscillations are localized near the bottom of density wells visible in Fig. 2 (left). If the plasma density in such a narrow well is modulated in the longitudinal direction due to, for example, the modulation instability, sub-luminal beam-driven modes can resonate with slow waveguide electromagnetic modes [5]. Then, these waveguide modes escape from the local density wells in the predominantly longitudinal direction via regular longitudinal inhomogeneity of plasma density on the scale of the magnetic field corrugation $(10 \mathrm{~cm})$. As to the high-frequency band $400 \div 800 \mathrm{GHz}$, Fig. 3 (spec- tral band in right side), on our opinion, is a result of the coalescence of two upper hybrid plasma waves into electromagnetic ones [6-8]. Since the sub-mm wave emission at the plasma density higher than $5 \times 10^{14} \mathrm{~cm}^{-3}$ is realized along the axis, we suppose that additional feature exists for longitudinal redirection of electromagnetic waves in the GOL-PET device. Note that the tendency to this redirection is qualitatively reproduced in theory [7].

\section{Conclusion}

It is found for the plasma density $(0.5 \div 1.0)$ $\times 10^{15} \mathrm{~cm}^{-3}$ that the radiation spectrum of the flux propagating along the axis of the beam-plasma system is mainly concentrated in two clearly distinct regions with the high level of spectral power density. The first region is located in the frequency interval $\mathrm{f}_{1}=0.15 \div 0.25 \mathrm{THz}$, the second one is in the interval $\mathrm{f}_{2}=0.4 \div 0.8 \mathrm{THz}$. The emission observed in the frequency interval $f_{1}$ has the power in the flux on a megawatt level and is interpreted as result of the linear conversion of the upper-hybrid branch of plasma oscillations to the electromagnetic radiation in regions of strong plasma density gradients. We interpret the emission in the interval $f_{2}$ as result of merging of these two plasma oscillations into the electromagnetic wave at a high level of plasma turbulence.

This work is supported by the Russian Foundation for Basic Research (grant 18-02-00232).

\section{References}

1. A. V. Arzhannikov, et al., // Transactions of Fusion Science and Technology, 2013, vol. 63, pp. 82-87.

2. I. A. Ivanov, et al., // AIP Conf. Proc., 2016, vol.1771, p.070009, doi.org/10.1063/1.4964233.

3. A.V. Arzhannikov, et al., // AIP Conf. Proc., 2016, v.1771, p.070004, doi.org/10.1063/1.4964228.

4. I.V. Timofeev, V.V. Annenkov and A.V. Arzhannikov // Phys. Plasmas 2015, vol.22, p.113109.

5. C.Miao, J.P.Palastro, and T.M. Antonsen // Physics of Plasmas, 2017, vol.24, p.043109, doi.org/10.1063/1.4981218.

6. I.V.Timofeev // Phys. Plasmas 2012, vol.19, p.044501.

7. A.V.Arzhannikov and I.V.Timofeev // Plasma Phys. Control. Fusion, 2012, vol.54, p.105004.

8. A. V. Arzhannikov et al., // IEEE Trans. Terahertz Sci. Technol., 2016, vol. 6, no 2, pp.245-252. 